\title{
Charge Dynamics and Spin Blockade in a Hybrid Double Quantum Dot in Silicon
}

\author{
Matias Urdampilleta, ${ }^{1, *}$ Anasua Chatterjee, ${ }^{1}$ Cheuk Chi Lo, ${ }^{1,2}$ Takashi Kobayashi, ${ }^{3}$ John Mansir, ${ }^{1}$ Sylvain Barraud, \\ Andreas C. Betz, ${ }^{5}$ Sven Rogge, ${ }^{3}$ M. Fernando Gonzalez-Zalba, ${ }^{5, \dagger}$ and John J. L. Morton ${ }^{1,2}$ \\ ${ }^{1}$ London Centre for Nanotechnology, University College London, London WC1H OAH, United Kingdom \\ ${ }^{2}$ Department of Electronic \& Electrical Engineering, University College London, \\ London WCIE 7JE, United Kingdom \\ ${ }^{3}$ Centre for Quantum Computation and Communication Technology, School of Physics, \\ University of New South Wales, Sydney NSW 2052, Australia \\ ${ }^{4}$ CEA, LETI, Minatec Campus, F-38054 Grenoble, France \\ ${ }^{5}$ Hitachi Cambridge Laboratory, J. J. Thomson Avenue, Cambridge CB3 OHE, United Kingdom
}

(Received 20 March 2015; published 27 August 2015)

\begin{abstract}
Electron spin qubits in silicon, whether in quantum dots or in donor atoms, have long been considered attractive qubits for the implementation of a quantum computer because of silicon's "semiconductor vacuum" character and its compatibility with the microelectronics industry. While donor electron spins in silicon provide extremely long coherence times and access to the nuclear spin via the hyperfine interaction, quantum dots have the complementary advantages of fast electrical operations, tunability, and scalability. Here, we present an approach to a novel hybrid double quantum dot by coupling a donor to a lithographically patterned artificial atom. Using gate-based rf reflectometry, we probe the charge stability of this double quantum-dot system and the variation of quantum capacitance at the interdot charge transition. Using microwave spectroscopy, we find a tunnel coupling of $2.7 \mathrm{GHz}$ and characterize the charge dynamics, which reveals a charge $T_{2}^{*}$ of $200 \mathrm{ps}$ and a relaxation time $T_{1}$ of $100 \mathrm{~ns}$. Additionally, we demonstrate a spin blockade at the inderdot transition, opening up the possibility to operate this coupled system as a singlet-triplet qubit or to transfer a coherent spin state between the quantum dot and the donor electron and nucleus.
\end{abstract}

DOI: 10.1103/PhysRevX.5.031024

\section{INTRODUCTION}

Quantum physics, when applied to computing, is projected to usher in a new computational paradigm with great potential applications in cryptography and simulation [1]. Most of the research effort has focused on the creation of the building blocks of quantum computing, called qubits: elements that have to be resistant to environmental disturbance in order to preserve the coherent information encoded in them. Many different media have been investigated to create such qubits, from superconducting devices [2] to atomlike spins in semiconductors [3]. In the last decade, silicon has attracted particular interest [4] because of its ability to create qubits out of implanted donor atoms [5], as well as the compatibility of a silicon quantum architecture with the microelectronics industry [6].

\footnotetext{
*m.urdampilleta@ucl.ac.uk

†mg507@cam.ac.uk
}

Published by the American Physical Society under the terms of the Creative Commons Attribution 3.0 License. Further distribution of this work must maintain attribution to the author(s) and the published article's title, journal citation, and DOI.
Subject Areas: Condensed Matter Physics, Nanophysics, Quantum Physics

Interest in donor-based spin qubits in silicon has been motivated by their exceptionally long electron spin coherence times, exceeding $1 \mathrm{~s}$ in isotopically enriched ${ }^{28} \mathrm{Si}$ [7]. Additionally, the donor electron spin can be a gateway to access the donor nuclear spin, which has longer coherence times [8], even at room temperature [9], and the potential to serve as a quantum memory [10]. Moreover, the single-shot read-out of single electron [11] and nuclear [12] spins, a milestone for donor-based quantum computing $[6,13,14]$, has recently been demonstrated in nanoelectronic silicon devices.

On the other hand, artificial atoms such as electrostatically defined quantum dots offer complementary advantages as qubits, notably in their tunability [15], flexible coupling geometries [16], and opportunities for fast electrical [17-20] and magnetic [21,22] control of spin. It is therefore attractive to investigate the possibility of hybrid architectures, which bring together the advantages of these two systems by coupling a quantum dot to a donor atom $[23,24]$. Such a double dot could take advantage of fast spin manipulations using gate voltage to form a hybrid singlet-triplet qubit coupled to the long-lived quantum memory offered by its nuclear spin. In addition, this hybrid architecture could be used to create spin buses with 
quantum-dot chains to mediate quantum information stored in donor qubits over long distances [25,26].

In this work, we present measurements taken on a silicon nanowire transistor indicating a coupled system formed by a single phosphorus atom and a quantum dot. We show that this hybrid system behaves as a double quantum dot, and we characterize the high-frequency admittance of the coupled system by rf gate-based sensing. Quantumcapacitance changes at the interdot charge transition (ICT) and microwave (MW) spectroscopy allow us to characterize the tunnel coupling and the charge dynamics. Finally, we demonstrate spin blockade effects between singlet and triplet states by applying a magnetic field that changes the resonator response at the ICT. These results demonstrate the potential of a donor-dot system as a new singlet-triplet qubit.

\section{CHARACTERIZATION OF THE DEVICE}

\section{A. Device fabrication and measurement setup}

The nanowire transistor device, sketched in Fig. 1(a), is fully compatible with the CMOS industry. It is fabricated from an silicon on insulator substrate composed of a 145-nm buried oxide layer and a 11-nm-thick silicon layer doped with phosphorus at a concentration of $5 \times 10^{17} \mathrm{~cm}^{-3}$. The doped silicon layer is etched to create a 200 -nm-long and 30 -nm-wide nanowire by means of deep-UV lithography. A 30-nm-wide wraparound top gate is defined using a $\mathrm{SiO}_{2}(0.8 \mathrm{~nm}) / \mathrm{HfSiON}(1.9 \mathrm{~nm})$ stack for the gate dielectric followed by $\mathrm{TiN}(5 \mathrm{~nm}) / \operatorname{poly}-\mathrm{Si}(50 \mathrm{~nm})$ as the top gate material. The self-aligned source and drain (not represented on the sketch) are formed by ion implantation after the deposition of 20-nm-thick $\mathrm{Si}_{3} \mathrm{~N}_{4}$ spacers (see Fig. S1).

Measurements are performed at the base temperature of an Oxford Instruments Triton 200 cryogen-free dilution refrigerator $(30 \mathrm{mK})$. High-sensitivity charge detection is achieved by radio-frequency reflectometry on a tank circuit composed of a surface-mounted inductance $(390 \mathrm{nH})$, a parasitic capacitance to ground $(0.75 \mathrm{pF})$, and the device capacitance between the transistor top gate and the channel [27]. Rf reflectometry is performed close to the resonance frequency $(294 \mathrm{MHz})$, with the input power set to $-85 \mathrm{dBm}$ and the reflected signal amplified by a low-noise cryogenic amplifier anchored at $4 \mathrm{~K}$. The signal is further amplified and demodulated at room temperature, as shown in Fig. 1(b). An on-board bias tee is used to apply both $\mathrm{dc}$ and $\mathrm{rf}$ voltages on the top gate. The undoped silicon substrate is activated by flashing a surface-mounted blue LED to generate free carriers, and it can then be used as a back gate [28].

\section{B. Charge stability diagram}

We first characterize the device charge stability diagram as a function of top-gate $\left(V_{\mathrm{tg}}\right)$ and back-gate voltages $\left(V_{\mathrm{bg}}\right)$. $\mathrm{Rf}$ reflectometry is used to detect a change in the sample impedance due to a dissipative or dispersive event
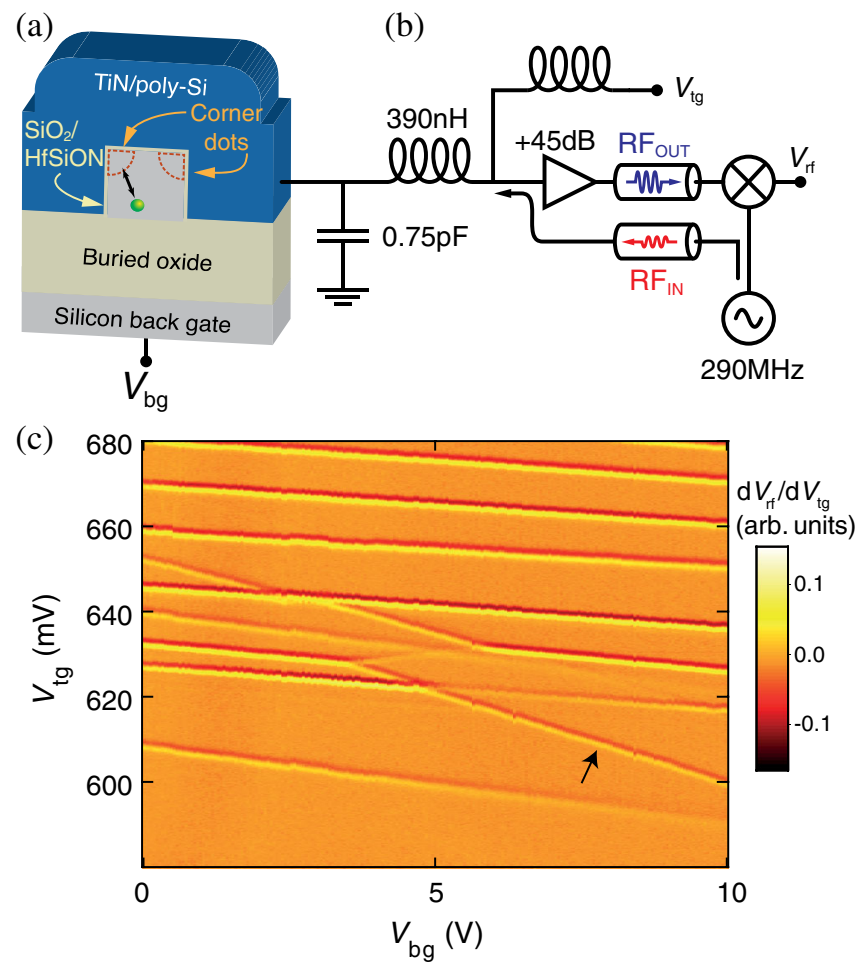

FIG. 1. (a) A sketch of the device, showing a cross section of the nanowire channel and wraparound gate, with quantum dots localized in the top corners of the nanowire and a dopant located deeper in the channel. For clarity, the source and drain are not represented on this sketch. (b) In our rf reflectometry measurements, an rf signal is sent through a directional coupler to a resonator made from the device capacitance, parasitic capacitance, and surface-mounted inductor. The reflected signal is amplified at low temperatures and demodulated using a reference signal to give $V_{\text {rf }}$. (c) The derivative of the demodulated resonator response as a function of $V_{\mathrm{tg}}$ and $V_{\mathrm{bg}}$ gives the charge stability diagram. Transitions with weak $V_{\text {bg }}$ dependence are attributed to changes in the charge occupancies of the two distinct (and uncoupled) corner dots. The charge transition indicated with a black arrow is attributed to a phosphorus atom in the bulk of the nanowire.

occurring below the top gate. As a consequence, it can probe a change of resistance due to, for instance, a charge tunneling between the source and a localized state $[27,29,30]$, or a change of quantum capacitance induced by a charge tunneling between two quantum dots [31,32]. Figure 1(c) presents the Gaussian-filtered numerical derivative of the reflected resonator signal amplitude as a function of $V_{\mathrm{tg}}$ and $V_{\mathrm{bg}}$. It shows a set of charge transitions with a small dependence on $V_{\mathrm{bg}}$ corresponding to single charge tunneling from the source or drain to quantum dots localized below the top gate. We attribute these charge transitions to the so-called corner quantum dots, formed in the top corners of the nanowire where the electric field is maximum, when the channel is in the subthreshold regime [33]. These dots have relatively large charging energies, 
$E_{c} \sim 18 \mathrm{meV}$, and a strong top-gate lever arm, $\alpha_{t g} \sim 0.85$, similar to what has been reported elsewhere $[27,33]$.

However, one single transition, indicated with a black arrow in Fig. 1(c), is more coupled to the back gate than the other charge transitions: This corresponds to a localized state lying further away from the nanowire-top-gate interface. Such subthreshold resonances are commonly attributed to single donors located in the channel [34,35]. This is likely to be the case in the present sample and in the one presented in Fig. S5 (see Ref. [36]), as the charging event occurs very close to the threshold $(650 \mathrm{mV})$ indicating an impurity state close to the conduction band edge, as expected for phosphorus atoms in silicon [37]. In addition, from the stability diagram and the back-gate or top-gate lever-arm ratio, we can place the impurity far below the surface, eliminating any interface charge trap or electron puddle, while at the same time, the number of bulk defects in the nanowire active region $(30 \mathrm{~nm} \times 30 \mathrm{~nm} \times 11 \mathrm{~nm})$ is a few orders of magnitude lower than the average number of dopants that were implanted (about 5 dopants in the channel). Finally, no subthreshold signatures were observed in any of the undoped devices (see Ref. [27]).

\section{CHARGE DYNAMICS}

\section{A. Interdot charge transition and quantum capacitance}

In Fig. 2(a) we focus on a particular region of interest in the charge stability diagram, showing the classic signature of a double quantum dot through the presence of an extra ridge at the intersection between the charge transitions of a corner dot and the donor. The charge number $(1,1)$ corresponds to one electron located on the corner dot and one at the donor site, and $(0,2)$ indicates that both electrons are on the donor. This charge assignment is deduced from two complementary measurements. First, we exploit the phase contrast of the reflected signal on the stability diagram (see Ref. [36], Fig. S2), to assign the two corner dots' charge occupancy. We deduce that the corner dot transition, seen in Fig. 2(a), corresponds to a transition from $(0,1)$ to $(1,1)$. Second, the charge transition shifts to higher gate voltage with the magnetic field (see Ref. [36], Fig. S3). This shift indicates that the Zeeman energy increases the donor chemical potential; as a result and in agreement with previous literature [38], we attribute the charge degeneracy to a $D^{0}: D^{-}$transition, where $D^{0}$ is the neutral donor and $D^{-}$the anion. Moreover, we observe this transition very close to the threshold, as expected [39].

We now investigate the coupling between the donor and one of the corner dots. The reflectometry signature of a charge tunneling between the donor and a corner dot is obtained by measuring the resonator response at an intersection between their charge transitions. The signal amplitude $A$ at the ICT can be shown to be a function of the quantum capacitance; its model is described in Ref. [40] and depicted in Figs. 2(b) and 2(c). When the energy levels
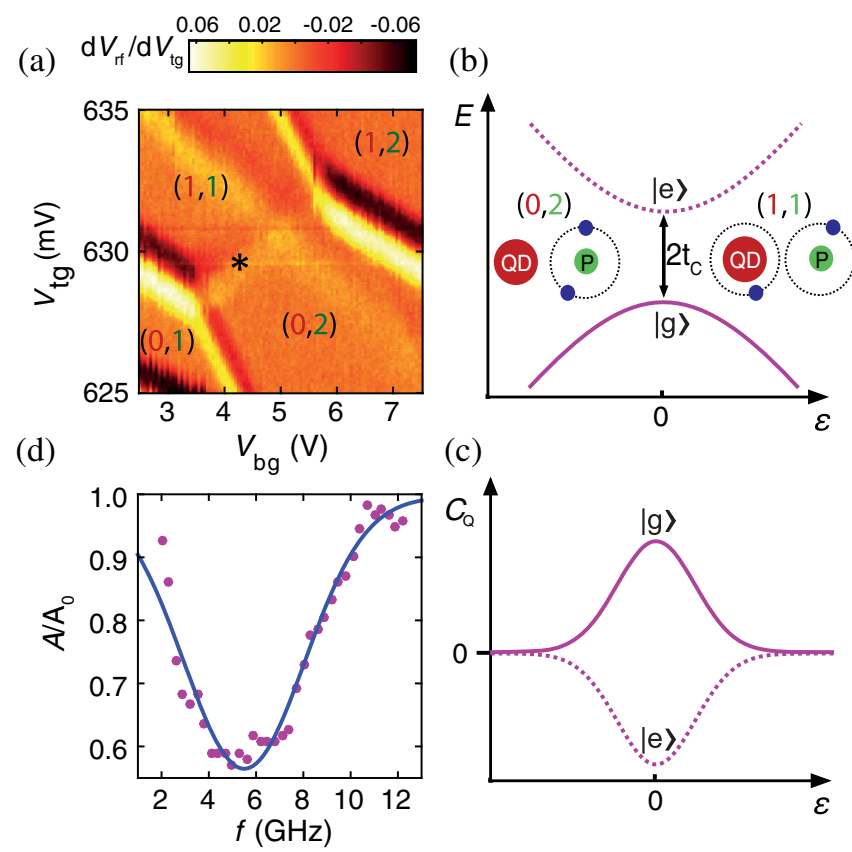

(c)

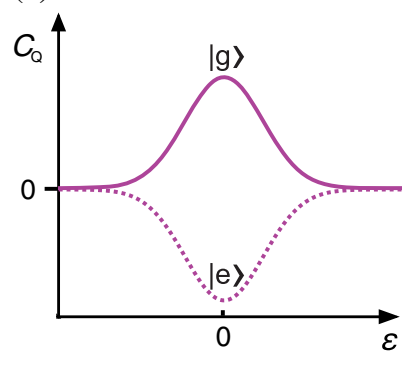

FIG. 2. (a) A close-up of the charge stability diagram showing the charge transition between a corner dot and a donor, with charge occupancies labeled as (corner dot, donor). The "asterisk" indicates the avoided level crossing sketched in (b) the energy levels of the hybrid double quantum dot, where the quantum dot and the $\mathrm{P}$ atom form bonding $|g\rangle$ and antibonding $|e\rangle$ states when the detuning $\epsilon$ is near zero. For simplicity, spin effects are omitted here and will be discussed later. For $|\epsilon| \gg 0$, the double dot has ionic-type wave functions, where the charges are localized on the dot or donor. (c) States $|g\rangle$ and $|e\rangle$ have opposite values of quantum capacitance, and hence, monitoring [panel (d)] the interdot charge transition (asterisk), as a function of applied microwave frequency, shows a reduction in signal amplitude upon resonance with the $|g\rangle:|e\rangle$ transition (see S4 for more details).

of the donor and the corner dot are brought into resonance, the tunnel coupling between the two quantum systems gives rise to a set of molecular orbitals: a bonding state and an antibonding state. The quantum capacitance is directly proportional to the curvature of the eigenenergies with respect to detuning [31],

$$
C_{q}=-(e \alpha)^{2} \frac{\partial^{2} E}{\partial \epsilon^{2}}
$$

where $\alpha$ is the coupling between the resonator and the double quantum dot, $E$ the eigenenergies, and $\epsilon$ the detuning energy. The quantum capacitance is then maximum for $\epsilon=0$, where the curvature of the eigenenergies is maximum. Notably, the quantum capacitances of the bonding state $|g\rangle$ and antibonding state $|e\rangle$ are of opposite sign.

\section{B. Tunnel coupling and charge dephasing time}

We exploit these quantum capacitance signatures to characterize the tunnel coupling between the donor and 
the corner dot, as well as the charge dynamics in the system, starting by performing MW spectroscopy using a local antenna coupled to the top gate. Figure 2(d) shows the relative change of signal, $A / A_{0}$, at the ICT as a function of the MW frequency, where $A_{0}$ is the signal amplitude without any MW applied. The MW excitation causes a fraction of the ground-state population to be promoted to $|e\rangle$, reducing the averaged quantum capacitance. As a result, the maximum change of signal occurs when the MW frequency matches the tunnel splitting, which in our case gives $\Delta=2 t_{c}=5.5 \mathrm{GHz}$. We use a Gaussian function to fit the data, from which we extract the charge coherence time $T_{2}^{*} \sim 200 \mathrm{ps}$, very similar to the one measured in a double-donor system in the same type of nanowire transistors [35]. The present dephasing rate is close to the tunnel splitting; therefore, implementation of a quantum protocol that uses coherent charge transfer to mediate spin information, such as coherent transfer by adiabatic passage (CTAP) [41], would have limited fidelity. However, while the tunnel splitting might be tunable in alternative device architectures [35], the coherence time may be improved either by going to lower temperatures (if it is limited by charge relaxation) or by improving the charge stability of the device (if it is charge noise limited).

\section{Charge relaxation}

To confirm that the coherence time is not limited by charge relaxation, we present a measurement of the charge relaxation time $T_{1}$ between the bonding and antibonding states. We use MW excitation to populate $|e\rangle$ and measure how fast it decays to the ground state. The relaxation time is expected to be short compared to our demodulator and voltage amplifier bandwidth (about a few MHz), making a transient measurement impossible in our case. Instead, we use a procedure developed by Petta et al. [42], where the MW excitation is chopped at some frequency $1 / \tau$ with a $50 \%$ duty cycle while time averaging the signal at the ICT. We define the charge polarization as $P=\left(P_{g}-P_{e}\right)$, with $P_{g}$ and $P_{e}$ the ground- and excited-state populations.

When the period $\tau$ is long compared to $T_{1}$, the timeaveraged polarization is $\langle P\rangle \approx 1 / 2\left(P_{\text {sat }}+P_{\text {th }}\right)$. This is because the polarization is, to a good approximation, at saturation, $P_{\text {sat }}$, during the first part of the cycle (MW on) and at thermal equilibrium, $P_{\text {th }}$, during the second part (MW off). When $\tau$ is short compared to $T_{1}$, the system has no time to relax to the ground state, and it is then at saturation throughout the cycle, giving $\langle P\rangle \approx P_{\text {sat }}$. Charge relaxation takes the system between these extremes, giving

$$
\langle P\rangle=\frac{\left(P_{\mathrm{sat}}+P_{\mathrm{th}}\right)}{2}+\left(P_{\mathrm{sat}}-P_{\mathrm{th}}\right) \frac{T_{1}\left(1-e^{-\tau / 2 T_{1}}\right)}{\tau} .
$$

In the present study, the normalized amplitude $A / A_{0}$ at the ICT is directly proportional to $\langle P\rangle / P_{\mathrm{th}}$; thus, $P_{\text {sat }} / P_{\text {th }}$ is obtained from the ICT signal under continuous MW
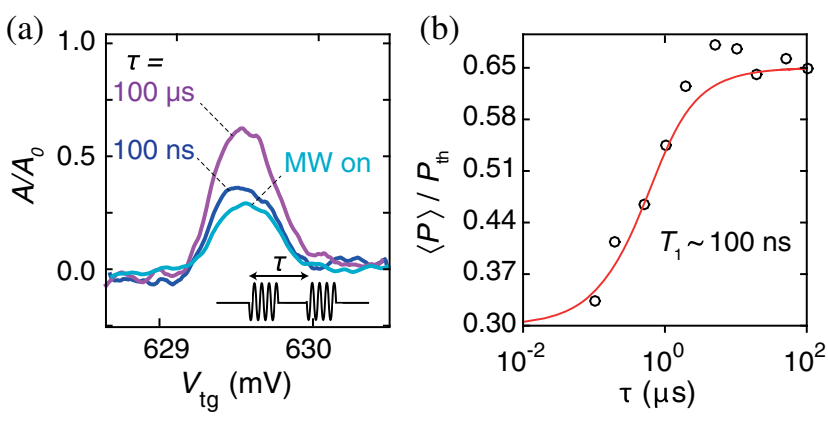

FIG. 3. (a) Interdot charge transitions measured under continuous resonant MW (light blue), and chopped MW at $10 \mathrm{MHz}$ (dark blue) and at $10 \mathrm{kHz}$ (purple). (b) Renormalized average charge-state population difference as a function of the chopping period $\tau$. The data are fitted using Eq. (2) to give the charge relaxation time $T_{1}$.

excitation [see Fig. 3(a)]. The power dependence of the saturation level is plotted in Ref. [36], Fig. S3. Also shown in Fig. 3(a) is the ICT under MW excitation chopped with 100-ns and 100- $\mu$ s time periods. As expected, the signal amplitude is greater when the charge has more time to relax.

The time-averaged polarization recorded for different values of $\tau$ is presented in Fig. 3(b) and is fitted to give $T_{1} \sim 100 \mathrm{~ns}$. Since $T_{1} \gg T_{2}^{*}$, charge coherence is not limited by charge relaxation. Moreover, the phononinduced dephasing rate in silicon is expected to be in the $\mathrm{MHz}$ range [43]. Therefore, background charge fluctuations or noise in the gate voltages are assumed to be the main dephasing sources as they induce fluctuations in both tunnel coupling and detuning energy [44].

\section{SPIN BLOCKADE}

We now investigate spin-related effects in the system, in order to demonstrate unambiguously that the ICT is of even parity. Figure 4(a) displays the ICT as a function of $V_{\mathrm{tg}}$ and the magnetic field, $B$. It shows that the reflectometry signal disappears with an increasing magnetic field and that, above about $1.2 \mathrm{~T}$, the variation of quantum capacitance at the ICT has completely vanished. This result may be explained as follows: (i) at $\epsilon=0$, as the magnetic field is increased, the population of the singlet state decreases while the population of the $T_{-}$triplet state increases (and eventually dominates for $g \mu_{B} B \gg k_{B} T$ ), and (ii) the triplet state has a linear dependence on detuning and hence a zero quantum capacitance signature according to Eq. (1). A similar response has been observed in an InAs double quantum dot coupled to a rf resonator [40] and has been theoretically investigated in Ref. [45].

The shift in the maximum amplitude position observed at a low field [see Fig. 4(c)] can then be understood by considering the detuning value at which the singlet and $T_{-}$states intersect, $\epsilon_{\mathrm{ST}}$, which shifts with magnetic field, in 

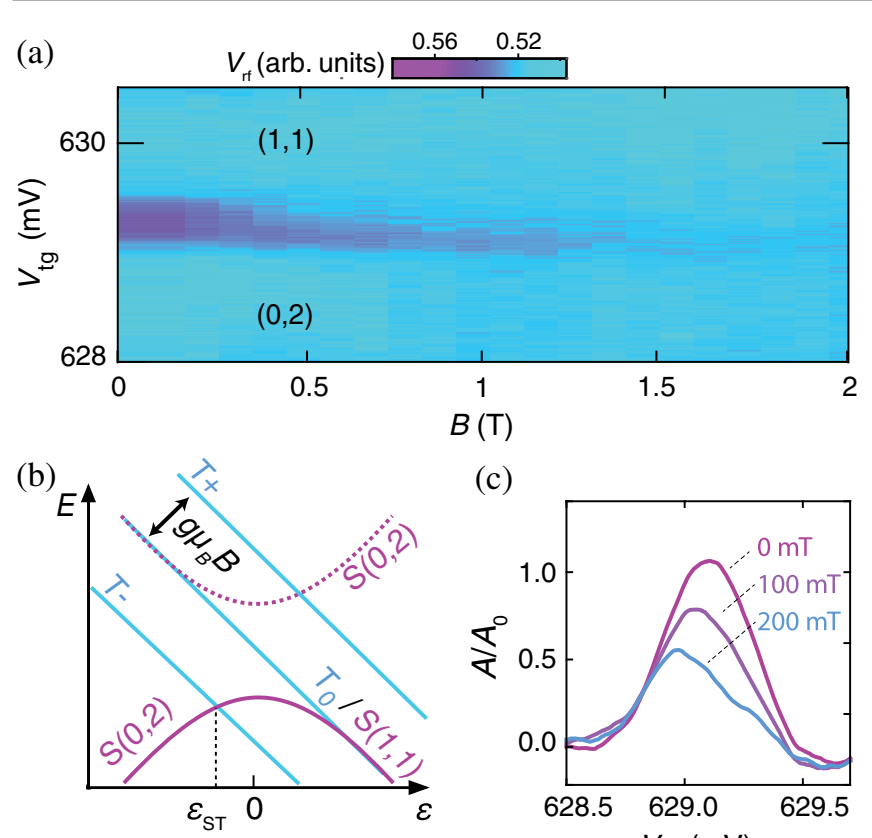

(c)

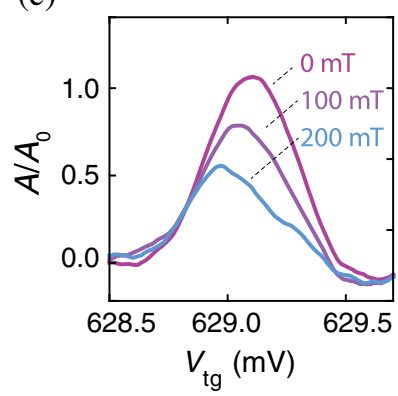

FIG. 4. (a) The quantum capacitance signature of the interdot charge transition (ICT) becomes strongly suppressed with increasing magnetic field, as the $T_{-}$triplet state becomes the ground state, as shown in the energy-level diagram [panel (b)] near the $(1,1)$ to $(0,2)$ transition in the presence of a magnetic field. $\epsilon_{\mathrm{ST}}$ denotes the value at which the singlet and $T_{-}$states intersect, and is responsible for the shift and asymmetry observed in (c), the ICT measured at $0 \mathrm{mT}, 100 \mathrm{mT}$ and $200 \mathrm{mT}$.

accordance with Fig. 4(b). For $\epsilon<\epsilon_{\mathrm{ST}}$, there is a singlet ground state and the quantum capacitance has a finite value, albeit one that decreases for more negative $\epsilon_{\mathrm{ST}}$. For $\epsilon>\epsilon_{\mathrm{ST}}$, there is a $T_{-}$ground state and the quantum capacitance vanishes. The asymmetric line shape observed is therefore a signature of the system with a significant population, which follows the ground state, even at the $\mathrm{S}: \mathrm{T}_{-}$intersection. This may be understood by the presence of an avoided level crossing combined with the fact that these measurements are inherently multipassage experiments. The mixing between these two states is generally induced by the magnetic-field gradient created by the nuclear spin bath [17]. In contrast, in this silicon hybrid double dot, the mixing should be dominated by the hyperfine interaction with the nuclear spin localized at the donor site. As a result, neglecting the Overhauser field created by the ${ }^{29} \mathrm{Si}$, the electron located on the donor is detuned by $\delta=A \mathbf{S} \cdot \mathbf{I}$, where $A$ is the hyperfine coupling constant, $\mathbf{S}$ the electron spin, and $\mathbf{I}$ the donor nuclear spin. In the case of phosphorus, $I=1 / 2$ and $A=117 \mathrm{MHz}$, the $\mathrm{S}(1,1)-\mathrm{T}_{0}$ coherent evolution at a very low exchange coupling could be driven at around $50 \mathrm{MHz}$. In the case of bismuth, $I=9 / 2$ and $A=1.48 \mathrm{GHz}$, the driving frequency should be even higher, ranging from $300 \mathrm{MHz}$ up to $3 \mathrm{GHz}$ depending on the nuclear spin state.

\section{OUTLOOK AND CONCLUSION}

In conclusion, we have investigated a hybrid double-dot system formed by a single donor and a corner dot in a single silicon nanowire transistor. Combining rf reflectometry with microwave spectroscopy has allowed us to determine a tunnel splitting $2 t_{c}=5.5 \mathrm{GHz}$ as well as to characterize the charge dynamics. The charge dephasing rate $1 / T_{2}^{*} \sim 5 \mathrm{GHz}$ is similar to the tunnel splitting and could be reduced by removing the nitride spacers in the device, which are known to possess a large trapped charge density. Also, the tunnel coupling might be tunable in a different architecture, with a split top gate for instance, such as that used in Ref. [35]. Furthermore, we have demonstrated a spin blockade at the interdot charge transition due to the presence of singlet and triplet states. The time evolution of such a singlet-triplet qubit should be governed by the donor nuclear spin, enabling controlled rotation gates when combined with NMR excitation [46]. Finally, exploiting the interdot exchange coupling would allow a SWAP operation between the corner dot and the donor atom spin state and, eventually, its storage in the donor nuclear spin using electronuclear double-resonance techniques.

\section{ACKNOWLEDGMENTS}

We thank Nicholas Lambert and Andrew Ferguson for discussions. The samples presented in this work were designed and fabricated by the TOLOP project partners. This research is supported by the EPSRC through the Materials World Network (EP/I035536/1), ARC Centre of Excellence for Quantum Computation and Communication Technology (Grant No. CE110001027), and UNDEDD (Project No. EP/K025945/1), as well as by the European Research Council under the European Community's Seventh Framework Programme (FP7/2007-2013) through Grant Agreements No. 279781 (ERC) and No. 318397. C. C. L. is supported by the Royal Commission for the Exhibition of 1851, and J. J. L. M. is supported by the Royal Society.

[1] E. Gibney, Quantum Computer Quest, Nature (London) 516, 24 (2014).

[2] M. H. Devoret and R. J. Schoelkopf, Superconducting Circuits for Quantum Information: An Outlook, Science 339, 1169 (2013).

[3] D. D. Awschalom, L. C. Bassett, A. S. Dzurak, E. L. Hu, and J. R. Petta, Quantum Spintronics: Engineering and Manipulating Atom-like Spins in Semiconductors, Science 339, 1174 (2013).

[4] F. A. Zwanenburg, A. S. Dzurak, A. Morello, M. Y. Simmons, L. C. L. Hollenberg, G. Klimeck, S. Rogge, S. N. Coppersmith, and M. A. Eriksson, Silicon Quantum Electronics, Rev. Mod. Phys. 85, 961 (2013).

[5] J. J. Pla, K. Y. Tan, J. P. Dehollain, W. H. Lim, J. J. L. Morton, D. N. Jamieson, A. S. Dzurak, and A. Morello, 
A Single-Atom Electron Spin Qubit in Silicon, Nature (London) 489, 541 (2012).

[6] B. E. Kane, A Silicon-Based Nuclear Spin Quantum Computer, Nature (London) 393, 133 (1998).

[7] A. M. Tyryshkin, S. Tojo, J. J. L. Morton, H. Riemann, N. V. Abrosimov, P. Becker, H.-J. Pohl, T. Schenkel, M. L. W. Thewalt, K. M. Itoh, and S. A. Lyon, Electron Spin Coherence Exceeding Seconds in High-Purity Silicon, Nat. Mater. 11, 143 (2012).

[8] M. Steger, K. Saeedi, M. L. W. Thewalt, J. J. L. Morton, H. Riemann, N. V. Abrosimov, P. Becker, and H.-J. Pohl, Quantum Information Storage for Over 180 s Using Donor Spins in a 28Si "Semiconductor Vacuum," Science 336, 1280 (2012).

[9] K. Saeedi, S. Simmons, J. Z. Salvail, P. Dluhy, H. Riemann, N. V. Abrosimov, P. Becker, H.-J. Pohl, J. J. L. Morton, and M. L. W. Thewalt, Room-Temperature Quantum Bit Using Ionized Donors in Silicon-28, Science 342, 830 (2013).

[10] J. J. L. Morton, A. M. Tyryshkin, R. M. Brown, S. Shankar, B. W. Lovett, A. Ardavan, T. Schenkel, E. E. Haller, J. W. Ager, and S. A. Lyon, Solid State Quantum Memory Using the 31P Nuclear Spin, Nature (London) 455, 1085 (2008).

[11] A. Morello, J. J. Pla, F. Zwanenburg, K. W. Chan, K. Y. Tan, H. Huebl, M. Möttönen, C. D. Nugroho, C. Yang, J. A. van Donkelaar, A. D. C. Alves, D. N. Jamieson, C. C. Escott, L. C. L. Hollenberg, R. G. Clark, and A. S. Dzurak, Single-Shot Readout of an Electron Spin in Silicon, Nature (London) 467, 687 (2010).

[12] J. J. Pla, K. Y. Tan, J. P. Dehollain, W. H. Lim, J. J. L. Morton, F. A. Zwanenburg, D. N. Jamieson, A. S. Dzurak, and A. Morello, High-Fidelity Readout and Control of a Nuclear Spin Qubit in Silicon, Nature (London) 496, 334 (2013).

[13] R. de Sousa, J. D. Delgado, and S. Das Sarma, Silicon Quantum Computation Based on Magnetic Dipolar Coupling, Phys. Rev. A 70, 052304 (2004).

[14] C. D. Hill, L. C. L. Hollenberg, A. G. Fowler, C. J. Wellard, A. D. Greentree, and H.-S. Goan, Global Control and Fast Solid-State Donor Electron Spin Quantum Computing, Phys. Rev. B 72, 045350 (2005).

[15] M. Veldhorst, J. C. C. Hwang, C. H. Yang, A. W. Leenstra, B. de Ronde, J. P. Dehollain, J. T. Muhonen, F. E. Hudson, K. M. Itoh, A. Morello, and A. S. Dzurak, An Addressable Quantum Dot Qubit with Fault-Tolerant Control Fidelity, Nat. Nanotechnol. 9, 981 (2014).

[16] M. D. Shulman, O. E. Dial, S. P. Harvey, H. Bluhm, V. Umansky, and A. Yacoby, Demonstration of Entanglement of Electrostatically Coupled Singlet-Triplet Qubits, Science 336, 202 (2012).

[17] S. Foletti, H. Bluhm, D. Mahalu, V. Umansky, and A. Yacoby, Universal Quantum Control of Two-Electron Spin Quantum Bits Using Dynamic Nuclear Polarization, Nat. Phys. 5, 903 (2009).

[18] J. R. Petta, A. C. Johnson, J. M. Taylor, E. A. Laird, A. Yacoby, M. D. Lukin, C. M. Marcus, M. P. Hanson, and A. C. Gossard, Coherent Manipulation of Coupled Electron Spins in Semiconductor Quantum Dots, Science 309, 2180 (2005).

[19] B. M. Maune, M. G. Borselli, B. Huang, T. D. Ladd, P. W. Deelman, K. S. Holabird, A. A. Kiselev, I. AlvaradoRodriguez, R.S. Ross, A. E. Schmitz, M. Sokolich,
C. A. Watson, M. F. Gyure, and A. T. Hunter, Coherent Singlet-Triplet Oscillations in a Silicon-Based Double Quantum Dot, Nature (London) 481, 344 (2012).

[20] E. Kawakami, P. Scarlino, D. R. Ward, F. R. Braakman, D. E. Savage, M. G. Lagally, M. Friesen, S. N. Coppersmith, M. A. Eriksson, and L. M. K. Vandersypen, Electrical Control of a Long-Lived Spin Qubit in a $\mathrm{Si} / \mathrm{SiGe}$ Quantum Dot, Nat. Nanotechnol. 9, 666 (2014).

[21] F. H. L. Koppens, C. Buizert, K. J. Tielrooij, I. T. Vink, K. C. Nowack, T. Meunier, L. P. Kouwenhoven, and L. M. K. Vandersypen, Driven Coherent Oscillations of a Single Electron Spin in a Quantum Dot, Nature (London) 442, 766 (2006).

[22] X. Hao, R. Ruskov, M. Xiao, C. Tahan, and H. Jiang, Electron Spin Resonance and Spin-Valley Physics in a Silicon Double Quantum Dot, Nat. Commun. 5, 3860 (2014).

[23] T. Schenkel, C. C. Lo, C. D. Weis, J. Bokor, A. M. Tyryshkin, and S. A. Lyon, A Spin Quantum Bit Architecture with Coupled Donors and Quantum Dots in Silicon, in Single Atom Nanoelectronic, edited by E. Prati and T. Shinada (Pan Stanford Publishing, Singapore, 2013), p. 255.

[24] M. F. Gonzalez-Zalba, D. Heiss, and A. J. Ferguson, A Hybrid Double-Dot in Silicon, New J. Phys. 14, 023050 (2012).

[25] M. Friesen, A. Biswas, X. Hu, and D. Lidar, Efficient Multiqubit Entanglement via a Spin Bus, Phys. Rev. Lett. 98, 230503 (2007).

[26] Y. Ping, J. H. Jefferson, and B. W. Lovett, A Coherent and Passive One Dimensional Quantum Memory, New J. Phys. 16, 103025 (2014).

[27] M. F. Gonzalez-Zalba, S. Barraud, A. J. Ferguson, and A. C. Betz, Probing the Limits of Gate-Based Charge Sensing, Nat. Commun. 6, 6084 (2015).

[28] B. Roche, B. Voisin, X. Jehl, R. Wacquez, M. Sanquer, M. Vinet, V. Deshpande, and B. Previtali, A Tunable, Dual Mode Field-Effect or Single Electron Transistor, Appl. Phys. Lett. 100, 032107 (2012).

[29] A. C. Betz, R. Wacquez, M. Vinet, X. Jehl, A. L. Saraiva, M. Sanquer, A. J. Ferguson, and M. F. Gonzalez-Zalba, Dispersively Detected Pauli Spin-Blockade in a Silicon Nanowire Field-Effect Transistor, Nano Lett. 15, 4622 (2015).

[30] J. I. Colless, A. C. Mahoney, J. M. Hornibrook, A. C. Doherty, H. Lu, A. C. Gossard, and D. J. Reilly, Dispersive Readout of a Few-Electron Double Quantum Dot with Fast rf-Gate Sensors, Phys. Rev. Lett. 110, 046805 (2013).

[31] K. D. Petersson, C. G. Smith, D. Anderson, P. Atkinson, G. A. C. Jones, and D. A. Ritchie, Charge and Spin State Readout of a Double Quantum Dot Coupled to a Resonator, Nano Lett. 10, 2789 (2010).

[32] S. J. Chorley, J. Wabnig, Z. V. Penfold-Fitch, K. D. Petersson, J. Frake, C. G. Smith, and M. R. Buitelaar, Measuring the Complex Admittance of a Carbon Nanotube Double Quantum Dot, Phys. Rev. Lett. 108, 036802 (2012).

[33] B. Voisin, V.-H. Nguyen, J. Renard, X. Jehl, S. Barraud, F. Triozon, M. Vinet, I. Duchemin, Y.-M. Niquet, S. de Franceschi, and M. Sanquer, Few-Electron Edge-State Quantum Dots in a Silicon Nanowire Field-Effect Transistor, Nano Lett. 14, 2094 (2014). 
[34] J. Verduijn, M. Vinet, and S. Rogge, Radio-Frequency Dispersive Detection of Donor Atoms in a Field-Effect Transistor, Appl. Phys. Lett. 104, 102107 (2014).

[35] E. Dupont-Ferrier, B. Roche, B. Voisin, X. Jehl, R. Wacquez, M. Vinet, M. Sanquer, and S. De Franceschi, Coherent Coupling of Two Dopants in a Silicon Nanowire Probed by Landau-Zener-Stückelberg Interferometry, Phys. Rev. Lett. 110, 136802 (2013).

[36] See Supplemental Material at http://link.aps.org/ supplemental/10.1103/PhysRevX.5.031024 for details about the measurements done on a second device showing similar behavior at the ICT.

[37] K. Y. Tan, K. W. Chan, M. Möttönen, A. Morello, C. Yang, J. Van Donkelaar, A. Alves, J. M. Pirkkalainen, D. N. Jamieson, R. G. Clark, and A.S. Dzurak, Transport Spectroscopy of Single Phosphorus Donors in a Silicon Nanoscale Transistor, Nano Lett. 10, 11 (2010).

[38] H. Sellier, G. P. Lansbergen, J. Caro, S. Rogge, N. Collaert, I. Ferain, M. Jurczak, and S. Biesemans, Transport Spectroscopy of a Single Dopant in a Gated Silicon Nanowire, Phys. Rev. Lett. 97, 206805 (2006).

[39] B. Voisin, M. Cobian, X. Jehl, M. Vinet, Y.-M. Niquet, C. Delerue, S. de Franceschi, and M. Sanquer, Control of the Ionization State of Three Single Donor Atoms in Silicon, Phys. Rev. B 89, 161404 (2014).
[40] M. D. Schroer, M. Jung, K. D. Petersson, and J. R. Petta, Radio Frequency Charge Parity Meter, Phys. Rev. Lett. 109, 166804 (2012).

[41] L. C. L. Hollenberg, A. D. Greentree, A. G. Fowler, and C. J. Wellard, Two-Dimensional Architectures for DonorBased Quantum Computing, Phys. Rev. B 74, 045311 (2006).

[42] J. R. Petta, A. C. Johnson, C. M. Marcus, M. P. Hanson, and A. C. Gossard, Manipulation of a Single Charge in a Double Quantum Dot, Phys. Rev. Lett. 93, 186802 (2004).

[43] Z. Shi, C. B. Simmons, D. R. Ward, J. R. Prance, R. T. Mohr, T. S. Koh, J. K. Gamble, X. Wu, D. E. Savage, M. G. Lagally, M. Friesen, S. N. Coppersmith, and M. A. Eriksson, Coherent Quantum Oscillations and Echo Measurements of a Si Charge Qubit, Phys. Rev. B 88, 075416 (2013).

[44] T. I. Itakura and Y. Tokura, Dephasing Due to Background Charge Fluctuations, Phys. Rev. B 67, 195320 (2003).

[45] A. Cottet, C. Mora, and T. Kontos, Mesoscopic Admittance of a Double Quantum Dot, Phys. Rev. B 83, 121311 (2011).

[46] R. Kalra, A. Laucht, C. D. Hill, and A. Morello, Robust Two-Qubit Gates for Donors in Silicon Controlled by Hyperfine Interactions, Phys. Rev. X 4, 021044 (2014). 